\title{
3D CFD ARC FAULT SIMULATION IN GAS-INSULATED SWITCHGEARS
}

\author{
F. ReicherT*, A. Petchanka \\ Siemens AG, Nonnendammallee 104, 13629 Berlin, Germany \\ * frank.reichert@siemens.com
}

\begin{abstract}
Arc fault processes can lead to strong damages in gas-insulated switchgears and have to be considered in the development process. In order to reduce test costs, the development of overpressure protection systems can be supported by CFD arc fault simulations. The paper deals with the modelling and simulation of arc fault processes in gas-insulated switchgears. The developed simulation tool takes into account a three-dimensional arc model and the opening of a rupture disc during the arc fault process. The influence of different insulating media as e.g. $\mathrm{SF}_{6}$, Air and $\mathrm{CO}_{2}$ on the arc fault process has been investigated. The simulation model has been validated by measured signals for pressure build-up and arc voltage.
\end{abstract}

Keywords: gas-insulated switchgear, internal arc, CFD arc fault simulation, modelling, validation.

\section{Introduction}

Internal arcs in gas-insulated switchgears can lead to strong damages of the switchgears and the surrounding volumes e.g. a substation. According to the standard [1], gas-insulated switchgears must be verified in type tests with respect to internal arcs. The effects of the respective arc fault process are the pressure increase of the insulating gas and the possible burn-through of the enclosure. In this context the effects of the arc fault process shall be limited to the appearance of a hole or a tear in the enclosure as it can be seen from the upper picture in Figure 1. Any kind of fragmentation is not allowed, see the lower picture in Figure 1.
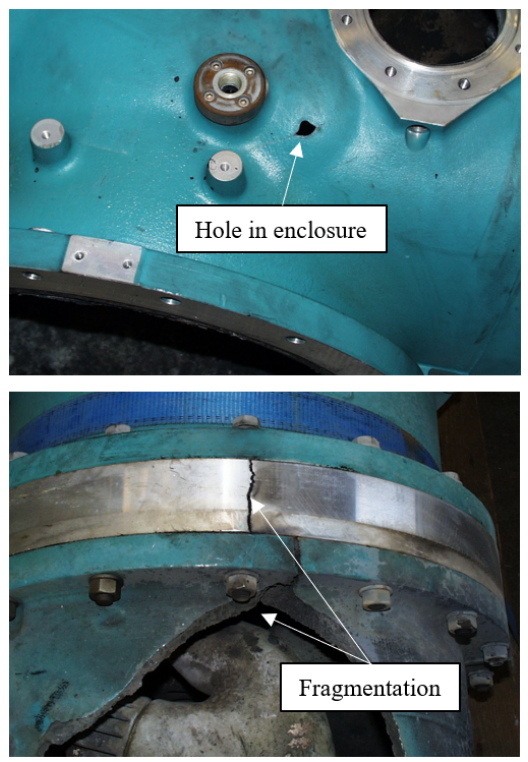

Figure 1. Visualization of different effects of the arc fault process.

The pressure build-up during the arc fault process can be controlled by the number, location, cross sec- tion, and response pressure of rupture discs. The technical design of such overpressure protection system can be supported by arc fault simulations.

There are many simulation approaches in the literature to simulate the arc fault process. The most simple approach considers the involved compartments by their effective volumes and describes the pressure increase in dependence on the thermal energy $Q$ as a part of the electrical energy $W_{\mathrm{el}}$ according to eq. (1), see e.g. [2]

$$
Q=k_{\mathrm{p}} \cdot W_{\mathrm{el}} \cdot
$$

In eq. (1) $k_{\mathrm{p}}$ corresponds to the heat transfer coefficient determining the fraction of the electrical arc energy, which directly results in a pressure rise. Such approach is based on assumptions for heat transfer coefficient and arc voltage as a part of the electrical energy.

In the present study, the developed CFD arc fault simulation tool that is based on ANSYS ${ }^{\circledR}$ Fluent software doesn't require an input for the heat transfer coefficient and the arc voltage, as the arc fault process is simulated using a three-dimensional arc model including a sub model describing the radiative transfer inside the arc and from the arc to the surrounding gas.

In Section 2 the basic aspects of the modelling are explained. The validation of the developed simulation tool is illustrated in Section 3 and the influence of the insulating medium on the arc fault process in Section 4. Finally, the conclusions are given in Section 5.

\section{Modelling}

\subsection{Sum current}

According to [3], it is possible to estimate the arc power $P_{\text {arc }}$ in a three-phase system by eq. (2)

$$
P_{\text {arc }}(t)=I_{\text {sum }}(t) \cdot U_{\text {arc }} \text {. }
$$


In eq. (2), $U_{\text {arc }}$ is the mean value of arc voltage and $I_{\text {sum }}(t)$ is the so-called sum current which corresponds to the sum of the three phase currents at any time $t$, see eq. (3)

$$
I_{\mathrm{sum}}(t)=0.5 \cdot\left(\left|i_{\mathrm{L} 1}(t)\right|+\left|i_{\mathrm{L} 2}(t)\right|+\left|i_{\mathrm{L} 3}(t)\right|\right) .
$$

Figure 2 shows an example for the three-phase currents and the resulting sum current calculated with eq. (3) over time.

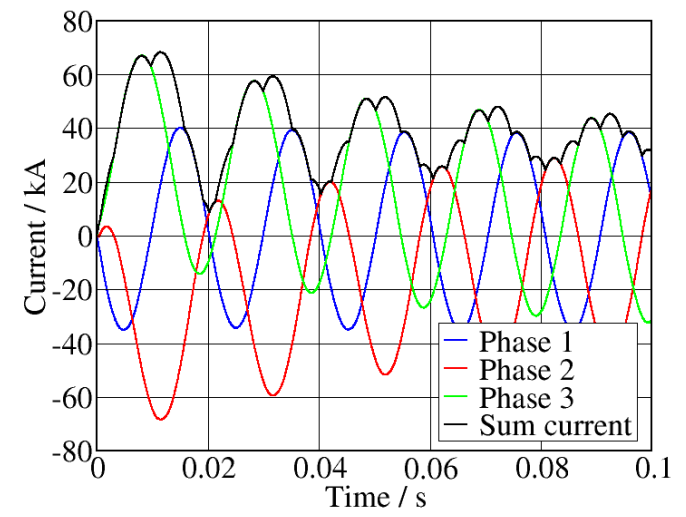

Figure 2. Three-phase currents and sum current.

\subsection{Arc fault model}

The implementation used for the arc fault in different insulating media is illustrated in Figure 3.

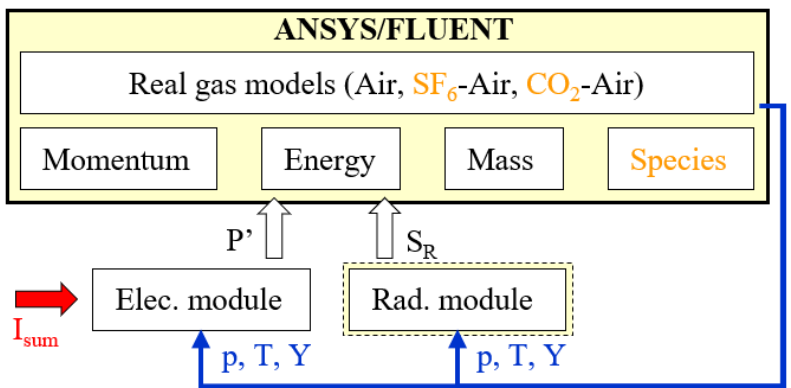

Figure 3. Link of conservation equations and sub models via source terms and scalar quantities.

The radiative transfer inside the arc fault and into its environment is reflected by the source term $S_{R}$ in the energy equation. This source term is calculated in the radiation module using the Discrete Ordinate Model.

The source term $P^{\prime}$ in the energy equation for the consideration of the Joule effect is calculated in the electric module. This source term is evaluated with a varying arc radius but with the assumption of a constant current density. The arc radius variation is controlled by the following formula

$$
R_{\mathrm{arc}}=\sqrt{\frac{I_{\mathrm{sum}}(t)}{\pi J_{\text {char }}}} .
$$

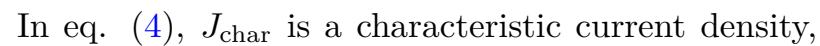
which has been derived from measurements. The assumption taken allows a more stable CFD arc simulation in $3 \mathrm{D}$ and bigger time step values.

To simulate the plasma flow, thermodynamic and transport properties of the insulating media must be provided to the ANSYS ${ }^{\circledR}$ Fluent solver. This is realized by real gas models for pure Air and the mixtures $\mathrm{SF}_{6}$ - Air and $\mathrm{CO}_{2}$ - Air, based on the assumption of local thermodynamic equilibrium (LTE). The scalar quantities pressure $p$, temperature $T$ and mass fraction $Y\left(\mathrm{SF}_{6}\right.$ or $\left.\mathrm{CO}_{2}\right)$ in case of $\mathrm{SF}_{6}-$ Air or $\mathrm{CO}_{2}-$ Air mixtures represent the input variables to the radiation module and the electric module.

\subsection{Operation of rupture disc}

As mentioned in Section 1 the pressure build-up during the arc fault process can be controlled by the opening of a rupture disc. The rupture disc opens at a pre-defined response pressure $p_{\text {open }}$. To consider this operation a macro has been developed. At the end of each time step the surface averaged pressure value $p_{\text {sim }}$ is estimated at the rupture disc wall and compared with $p_{\text {open }}$. If $p_{\text {sim }}$ exceeds $p_{\text {open }}$ the rupture disc wall is removed and the vessel volume is connected to the surroundings.

\section{Validation}

The 3D CFD arc fault simulation tool has been initially validated using a test arrangement investigated in [4]. The corresponding measurements have been performed in a closed test vessel filled with Air, $\mathrm{CO}_{2}$ or $\mathrm{SF}_{6}$ at different filling pressure. Figure 4 shows the current curve used in the simulations and the comparison of measured and simulated pressure within the test vessel for a filling pressure of 1 bar (absolute value).

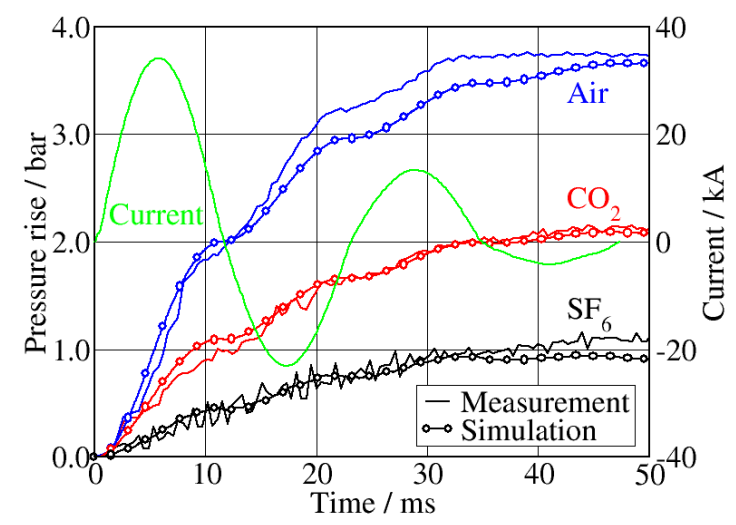

Figure 4. Pressure rise for different insulating media.

In addition, the 3D CFD arc fault simulation tool has been validated using a type test of a gas-insulated switchgear filled with Air. Figure 5 shows the discretized vessel without the surrounding solution domain. Using $I_{\text {sum }}(t)$ depicted in Figure 2 the arc 


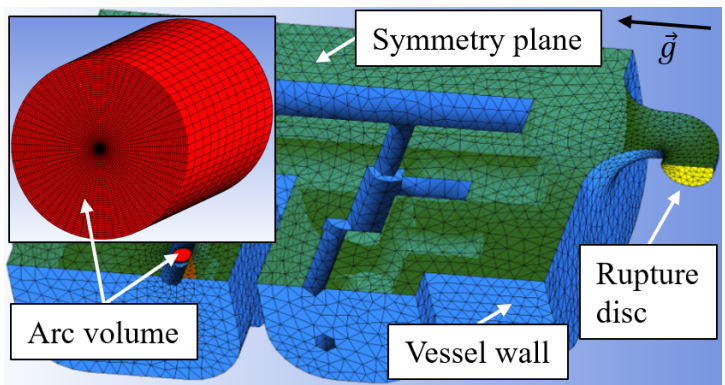

Figure 5. Half model of the regarded vessel with discretized arc volume.

fault process has been simulated for an arcing time of $0.5 \mathrm{~s}$ including the opening of the rupture disc and the following outflow of hot Air to the surroundings. For validation purposes concerning the type test, Figure 6 shows the comparison of measured and simulated arc voltage. In contrast to the oscillating arc voltages from the measurement the simulated arc voltage is more or less constant following the input by the sum current. The comparison with respect to the pres-

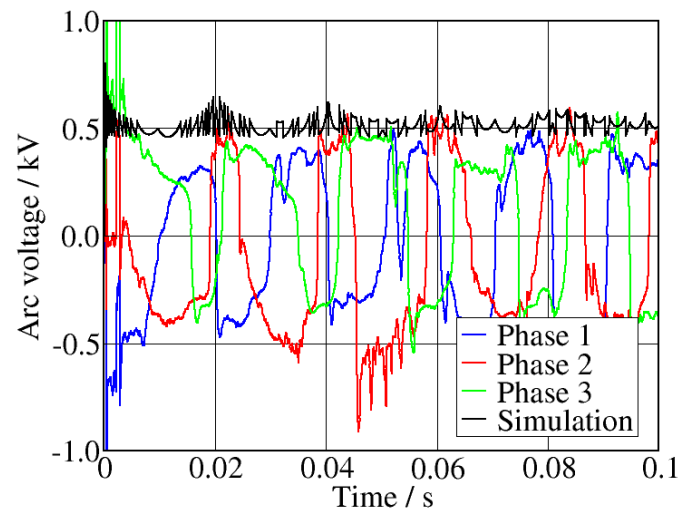

Figure 6. Comparison of measured and simulated arc voltage.

sure build-up within the gas-insulated switchgear is illustrated in Figure 10.

Despite the simplifications in arc modelling, the comparison according to Figure 4, 6 and 9 demonstrates a good agreement between measurement and simulation validating the $3 \mathrm{D}$ CFD arc fault simulation tool.

\section{Influence of insulating medium}

The illustrated CFD arc fault simulation tool allows to investigate the influence of different insulating media on the arc fault process.

Initially this influence should be demonstrated using the example of the arc fault process in the closed vessel from [4]. As it can be seen from Figure 5, the highest pressure build-up occurs in case of Air followed by $\mathrm{CO}_{2}$ and $\mathrm{SF}_{6}$. This behaviour is typical in case of a closed vessel and primarily a result of the arc heat capacity which can be calculated by the following formula

$$
C_{\text {arc }}\left(t^{\prime}\right)=\frac{\int_{0}^{t^{\prime}} P_{\text {arc }}(t) \mathrm{d} t}{\Delta T^{\prime}} .
$$

$C_{\text {arc }}$ is a function of transferred arc power $P_{\text {arc }}$ and resulting temperature change $\Delta T^{\prime}=T_{\text {arc }}\left(t^{\prime}\right)-T_{\text {init }}$. The comparison of the arc heat capacity for the three regarded insulating media is depicted in Figure 7. As

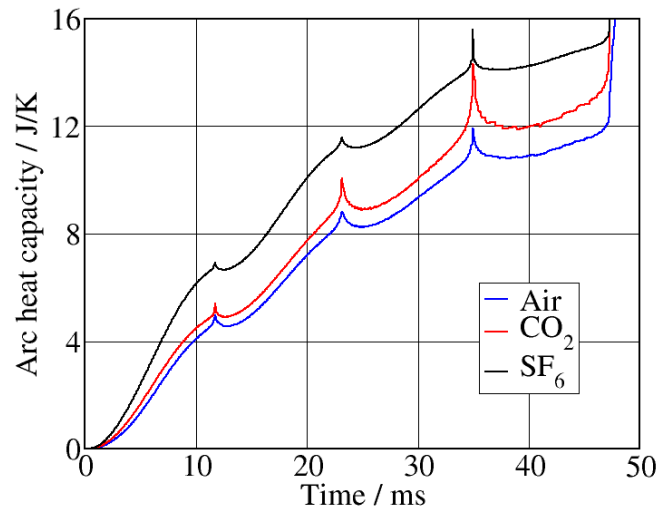

Figure 7. Comparison of simulated arc heat capacities.

it can be seen from Figure 7, Air features the smallest arc heat capacity leading to the highest pressure buildup depicted in Figure 4. Due to the significantly larger arc heat capacity of $\mathrm{SF}_{6}$, the corresponding pressure build-up is considerably lower. $\mathrm{CO}_{2}$ is in between.

According to eq. (5), the arc heat capacity depends on the transferred arc power and consequently on the arc voltage. Figure 8 shows the simulated arc voltages for the three regarded insulating media in comparison. The tooth-saw shape of the arc voltages

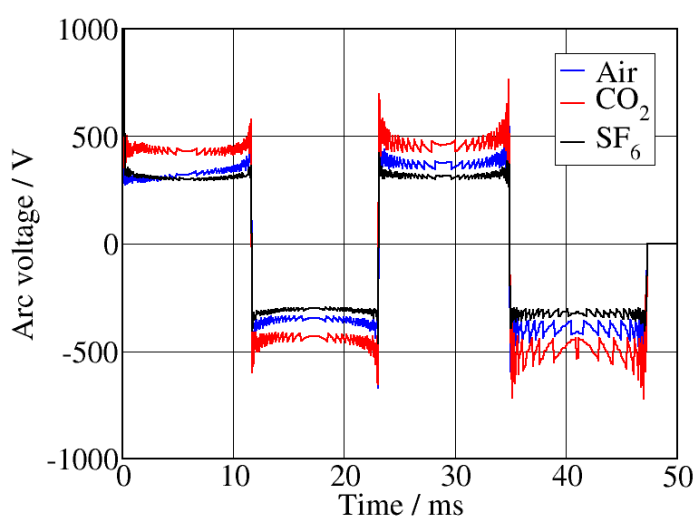

Figure 8. Comparison of simulated arc voltages.

is a result of the utilization of eq. (4) to control the arc radius variation in the discretized arc volume (see Figure 5). According to Figure 8, $\mathrm{CO}_{2}$ features the highest arc voltage followed by Air and $\mathrm{SF}_{6}$. This behaviour corresponds to the measurement results from [4].

The influence of the insulating media on the arc fault process should be explained in more detail using 
the type test highlighted in the previous section. For this purpose, the arc fault process has been additionally simulated with $\mathrm{CO}_{2}$ and $\mathrm{SF}_{6}$ under the same conditions for e.g. current input, filling pressure and response pressure of rupture disc. The operation of the rupture disc and the following outflow of hot gas to the surroundings influences the arc fault process significantly and changes the behaviour concerning the pressure build-up within the gas-insulated switchgear, see Figure 9. As long as the rupture disc is closed, the

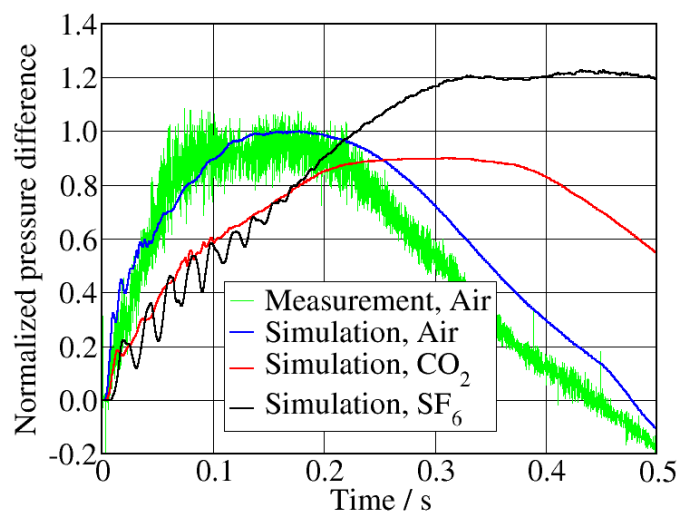

Figure 9. Pressure build-up in the gas-insulated switchgear (normalized to the maximum value in the simulation with Air).

arc fault process is mainly influenced by the arc heat capacity of the respective gas and the arc voltage as in the process in the closed vessel from [4]. After the opening of the rupture disc, the outflow of the hot gas is mainly influenced by the speed of sound, which depends inversely proportional on the gas density. As the initial pressure is the same, the gas density differs very strongly in the compared simulation variants. In case of $\mathrm{SF}_{6}$, the highest gas density exists and the hot gas flows out with the lowest speed of sound, see Figure 10. The highest values for the speed of sound

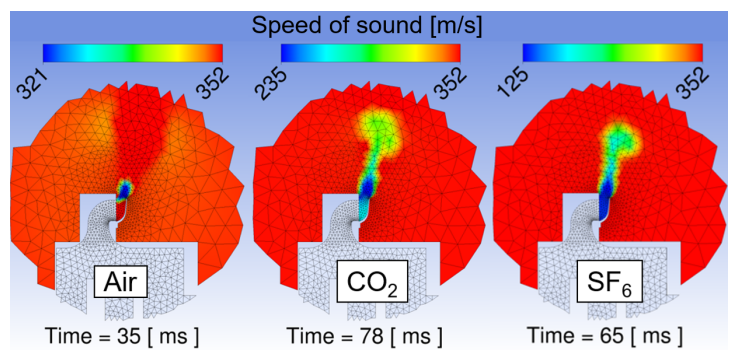

Figure 10. Contour plot of speed of sound in the symmetry plane about $5 \mathrm{~ms}$ after opening of rupture disc.

occur in case of Air. $\mathrm{CO}_{2}$ lies in between. Regarding the described differences in arc heat capacity, arc voltage and speed of sound, the pressure curves in Figure 9 can be explained. In case of Air, the arc fault process is connected with the steepest pressure rise but by the enforced outflow of hot gas, the maximum of pressure is reached very fast followed by a strong drop of pressure. In case of $\mathrm{SF}_{6}$, the pressure rises much slower compared to Air but the outflow of the hot gas is affected by the low speed of sound leading to a higher maximum of pressure. The lowest values for the maximum pressure are reached in case of $\mathrm{CO}_{2}$.

\section{Conclusions}

The present paper focuses on the basics of a $3 \mathrm{D}$ CFD arc fault simulation tool which can be used in the development of overpressure protection systems for gas-insulated switchgears.

The illustrated tool has been validated by measured signals for pressure build-up and arc voltage and allows to investigate the influence of different insulating media on the arc fault process. In this paper, the three insulating media Air, $\mathrm{CO}_{2}$ and $\mathrm{SF}_{6}$ have been considered.

It has been clarified, that in case of a closed vessel the highest pressure rise occurs in Air followed by $\mathrm{CO}_{2}$ and $\mathrm{SF}_{6}$. This behaviour results mainly from the corresponding arc heat capacities.

In case of a gas-insulated switchgear including a overpressure protection system, the pressure buildup within the vessel is also mainly influenced by the arc heat capacity of the respective gas, as long as the rupture disc is closed. After the opening of the rupture disc, the outflow of the hot gas is mainly influenced by the speed of sound of the gas and hence by the density of the insulatig medium within the vessel. In the present paper, the same initial pressure within the vessel has been considered in the $3 \mathrm{D}$ CFD arc fault simulation for all regarded insulating media. Thus the highest pressure build-up has been simulated for $\mathrm{SF}_{6}$ followed by Air and $\mathrm{CO}_{2}$.

\section{Acknowledgements}

The authors are grateful to Pierre Freton, Jean-Jacques Gonzalez, Yann Cressault and Philippe Teulet from the University Paul Sabatier in Toulouse for their support and participation in the developments of numerous submodels and for providing of thermodynamic, transport and radiation properties.

\section{References}

[1] High-voltage switchgear and controlgear - Part 203: Gas-insulated metal-enclosed switchgear for rated voltages above 52 kV. IEC 62271-203, 2011.

[2] N. Uzelac et al. Tools for the simulation of the effects of the internal arc in transmission and distribution switchgear. Cigre, Technical Brochures, 602, WG A3.24, 2014.

[3] X. Zhang. Modellierung der Auswirkungen von Störlichtbögen in elektrischen Anlagen. Postdoctoral thesis. RWTH Aachen, 2002.

[4] S. Wetzeler et al. Influence of insulating gas on pressure rise in electrical installations due to internal arcs. 20th International Conference on Gas Discharges and their Applications, pages 442-445, 2014. 